\title{
No linealidad y asimetría en el proceso generador del Î́ndice Ibex35
}

\author{
PAZ RICO BELDA \\ Universidad de Valencia, Facultad CC.EE., Avda. de los naranjos, s/n, 46022 Valencia, España. E- \\ mail: paz.rico@uv.es
}

\begin{abstract}
RESUMEN
El trabajo analiza el comportamiento del Ibex35, durante el período que abarca desde enero de 1999 a diciembre de 2011, con el objetivo de comprobar si sigue un proceso diferente al paseo aleatorio, de tal forma que su rendimiento no se caracteriza por ser ruido blanco y resulta, en contra de lo que implica la hipótesis de los mercados eficientes, predecible. Para ello se va a tener en cuenta la posibilidad de que el proceso generador del rendimiento sea no lineal y se va a especificar un modelo STAR-APARCH, que permite un comportamiento no lineal, tanto de la media como de la varianza condicional. Los resultados empíricos evidencian que el comportamiento del Ibex35 sigue un proceso no lineal y asimétrico, tanto en la media como en la varianza condicional, rechazándose el cumplimiento de la versión débil de la hipótesis de los mercados eficientes.
\end{abstract}

Palabras clave: Mercados eficientes, no linealidad, asimetría, media condicional, varianza condicional, modelos autorregresivos con umbral.

\section{Nonlinearity and Asymmetry in the Generator Process of Ibex35 Index}

\begin{abstract}
This paper analyzes the behaviour of Ibex35 from January 1999 to December 2001, in order to check if it follows a different process from random walk so its return is not a white noise and it can be predictable, against the efficient market hypothesis. For that, a nonlinear generating process of return will be considered and a STAR-APARCH model will be specified. This model allows a nonlinear behaviour in the conditional mean and in the conditional variance. The empirical results show that the Ibex35 follows a nonlinear and asymmetric process, both in the conditional mean as in the conditional variance, so the weak-version of efficient market hypothesis is rejected.
\end{abstract}

Keywords: Efficient Markets, Nonlinearity, Asymmetry, Conditional Mean, Conditional Variance, Threshold Autoregressive Models.

Clasificación JEL: G14, C22, C53

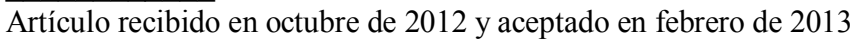

Artículo disponible en versión electrónica en la página www.revista-eea.net, ref. ə-31204 


\section{INTRODUCCIÓN}

La hipótesis de los mercados eficientes (HME) considera que el precio de un activo refleja el valor de mercado de los flujos futuros de la empresa y, además, cada activo se valora correctamente utilizando toda la información disponible. En consecuencia, el precio de los activos incorpora inmediata y adecuadamente toda la nueva información disponible. La versión débil de la HME argumenta que las variaciones de los precios son impredecibles. Por tanto, la mejor predicción para el precio de los activos es el valor corriente, por lo que el precio de los activos sigue el denominado paseo aleatorio y su rendimiento el de ruido blanco ${ }^{1}$.

Desde el trabajo seminal de Fama (1970), los trabajos para confirmar o rechazar la HME han sido muy numerosos ${ }^{2}$, pero a pesar de la extensa literatura no se puede obtener una conclusión definitiva. Para Torrero (2011) es el tópico más debatido en las finanzas y el abanico de opiniones va desde el incondicional apoyo de Jensen (1978), considerando que no hay ninguna proposición en economía que tenga un soporte de evidencia empírica más sólida, al fuerte rechazo de Shiller (1984), que la consideró uno de los mayores errores de la historia del pensamiento económico.

Recientemente muchos economistas han visto en esta hipótesis una de las causas de la actual crisis financiera. Torrero (2011) recoge la opinión de algunos de ellos, entre los que destaca Grantham (2010), para quien no hay duda de la responsabilidad que ha jugado la HME en la crisis financiera mundial de finales de 2007. Este autor considera que sin esta hipótesis "habríamos reconocido la disfunción de los mercados estableciendo más controles, que hubieran ayudado a limitar la salvaje expansión de las finanzas". Por su parte, Stiglitz (2010) argumenta que "si la hipótesis fuera cierta no existirían las burbujas. La experiencia de los excesos en las valoraciones que han conducido al desastre actual no puede ser más contundente". Asimismo, para James (2009) las crisis financieras son una prueba del incumplimiento de la HME: "De acuerdo con los mercados eficientes los precios de las acciones reflejan adecuadamente toda la información pública disponible de cada título, los cuales no cambiarán si no llega nueva información. Los pánicos en los cuales los precios cambian abruptamente no pueden ser fácilmente explicados según esta hipótesis, especialmente si el pánico no ha generado nueva información. En consecuencia, las crisis financieras han sido una fuente de críticas de los mercados eficientes". Para Woolley (2010) la influencia de la HME en las finanzas ha sido extraordinaria y su amplia aceptación explica en buena medida la crisis financiera actual.

\footnotetext{
${ }^{1}$ Samuelson (1965) demostró que en un mercado eficiente los cambios en el precio de los activos son impredecibles si incorporan toda la información disponible en el mercado.

${ }^{2}$ Ver Sewell (2011) que hace un repaso exhaustivo de la historia sobre la HME a lo largo del tiempo.
} 
Para este autor, "los banqueros centrales, al tener confianza en las cotizaciones de los mercados, tendieron a creer que las burbujas, bien no existían, bien eran beneficiosas para la economía, o no podían detectarse. La consecuencia fue justificar la no intervención. El escaso rigor de los reguladores descansaba en la confianza de la teoría y en la capacidad autorreguladora de la competencia". Por el contrario, Ball (2009) argumenta que el colapso de Lehman Brothers y de otras instituciones, lejos de ser el resultado de una excesiva fe en la HME, refleja la falta de atención a las enseñanzas de la hipótesis. Para este autor, la creencia equivocada de que los precios de los activos crecían constantemente se encuentra en la base de la reciente crisis.

De la extensa literatura empírica se obtiene resultados mixtos, de tal forma que entre los trabajos que evidencian que el precio de los activos no sigue un paseo aleatorio, y por tanto, la HME no es válida caben destacar De Bondt y Thaler (1985), Poterba y Summer (1988), Lo y MacKinlay (1988), Fama y French (1988), Urrutia (1995), Grieb and Reyes (1999), Chaudhuri y Wu (2003), Bali et al., (2008), Lee et al., (2010) y Kim et al., (2011). Entre los trabajos que muestran evidencia empírica de que la HME se cumple se pueden citar Chan et al., (1997), Choudhry (1997), Huber (1997), Liu et al., (1997), Kawakatsu y Morey (1999) y Narayan (2006).

Asimismo los trabajos que analizan distintos mercados bursátiles, tales como Worhtington y Higgs (2004), Kim y Shamsuddin (2009) y Borges (2011), tampoco obtienen conclusiones definitivas puesto que encuentran evidencia heterogénea, esto es, mercados que son eficientes y otros en los que la HME no se cumple.

Por lo que respecta a nuestro país, la evidencia empírica tampoco es concluyente. Así, Pérez-Rodríguez y Torra (2003) y Pérez-Rodríguez et al., (2005) obtienen que el Ibex35 se aparta ligeramente de la conducta de paseo aleatorio y evidencian la posibilidad de que los rendimientos bursátiles del Ibex35 se pueden predecir utilizando modelos no lineales. Por su parte, Worhington y Higgs (2004) encuentran que los rendimientos presentan correlación serial negativa, por lo que los precios de los activos no siguen un comportamiento de paseo aleatorio, mientras que para Kim y Shamsuddin (2009) y Borges (2011) la evidencia obtenida les lleva a concluir que el mercado bursátil español es eficiente.

Por otro lado, muchas investigaciones no solo han cuestionado el cumplimiento de la HME y que, por tanto, el modelo paseo aleatorio sea un proceso que describa razonablemente el comportamiento que sigue el precio de los activos, sino que también se han cuestionado que los modelos lineales sirvan para describir el comportamiento de tales precios ${ }^{3,4}$. Para Narayan (2006) la razón de

\footnotetext{
${ }^{3}$ Una relación de citas bibliográficas al respecto puede encontrarse en Pérez-Rodríguez y Torra (2003) y en Narayan (2006).
} 
no obtener una conclusión definitiva sobre el cumplimiento o no de la HME se debe al hecho de que los precios de los activos bursátiles se caracterizan por seguir procesos no lineales. Por su parte, Lim et al., (2008), Lim (2009) y Lim y Brooks (2009) muestran la existencia de dependencia serial no lineal en los rendimientos, llegando a considerar la no linealidad como un hecho estilizado del comportamiento de los rendimientos.

El objetivo del trabajo es contribuir a esclarecer si el mercado de valores español es un mercado eficiente. Para ello se va analizar el comportamiento estocástico del índice bursátil Ibex35, lo que permitirá comprobar si sigue un proceso diferente al paseo aleatorio y, por tanto, su rendimiento no se caracteriza por ser ruido blanco y resulta, en contra de lo que implica la HME, predecible. En este trabajo se va a tener en cuenta la posibilidad de que el proceso generador de los rendimientos sea no lineal, considerando un modelo self-exciting threshold autoregressive (STAR) de tres regímenes, con heterocesdasticidad condicional autoregresiva y asimétrica ${ }^{5}$. De esta forma, el modelo va a permitir tener en cuenta el posible comportamiento no lineal tanto de la media como de la varianza condicional de los rendimientos.

La estructura del trabajo es como sigue: tras esta introducción se analizan, en el apartado segundo, los datos. A continuación, en el apartado tercero se expone la metodología econométrica utilizada y se presenta la evidencia empírica obtenida. Finalmente, en el apartado cuarto, se recogen las principales conclusiones del trabajo.

\section{ANÁLISIS DE LOS DATOS}

En este trabajo se utilizan los datos de cierre diario del índice Ibex 35 de los días en los que existe mercado, durante el período comprendido entre el 4 de enero de 1999 y el 30 de diciembre de 2011, lo que da lugar a una muestra de 3.285 observaciones diarias ${ }^{6}$. Los datos han sido obtenidos del Boletín Estadístico del Banco de España. La serie se ha transformado en logaritmos, de tal forma que su variación proporciona el rendimiento entre dos días consecutivos de mercado. En concreto, se ha calculado el rendimiento diario para el día $t\left(R_{t}\right)$ de la siguiente forma:

\footnotetext{
${ }^{4}$ Las correcciones que hace el mercado de las desviaciones del precio de su valor teórico pueden no ser proporcionales a su tamaño, de tal forma que grandes desviaciones pueden tener un impacto diferente al producido por pequeñas desviaciones. Asimismo, la respuesta de los mercados puede ser asimétrica, de tal forma que los shocks negativos pueden tener un mayor impacto que los positivos de igual magnitud.

${ }^{5}$ En concreto, la heteroscedaticidad APARCH (Asymmetric power autoregressive condicional heteroskedasticity).

${ }^{6}$ El período comprendido entre el 2 de enero y el 31 de mayo de 2012 se reserva para analizar la capacidad predictiva del modelo fuera de la muestra.
} 


$$
R_{t}=100 x\left(\log I_{t}-\log I_{t-1}\right)
$$

donde $\log I_{t}$ es el logaritmo del índice Ibex35 en el día t.

Siguiendo a Rashid y Ahmad (2008) y Cao y Tsay (1992), la volatilidad del rendimiento diario se estima utilizando la siguiente expresión ${ }^{7}$ :

$$
\sigma_{t}=\sqrt{(\pi / 2)}\left|R_{t}-\mu\right|
$$

donde $\mathrm{R}_{\mathrm{t}}$ es el rendimiento diario para el día t y $\mu$ es la media de la serie.

La Tabla 1 recoge los estadísticos descriptivos del Ibex35 y de su rendimiento. Como puede observarse, el estadístico Bera-Jarque indica que ninguna de las series sigue una distribución normal. En la serie del rendimiento se observa que su distribución presenta una ligera asimetría hacia la derecha, pero es claramente leptocúrtica, puesto que el coeficiente de curtosis es mayor que tres, lo que significa que la distribución es más apuntada que la distribución normal y, por tanto, registra una mayor concentración de valores en torno al valor promedio. Este resultado, que también se observa en otros índices bursátiles $^{8}$, tiene una implicación importante puesto que las distribuciones leptocúrticas se caracterizan por presentar colas más gruesas que la distribución normal, haciendo más probable la "aparición" de rendimientos en los extremos de las colas.

Tabla 1

Estadísticos descriptivos

\begin{tabular}{lcccccccccc}
\hline & \multicolumn{3}{c}{ Media $\begin{array}{c}\text { Desviación } \\
\text { típica }\end{array}$} & \multicolumn{4}{c}{ Asimetría Curtosis } & Máximo & Mínimo & \multicolumn{3}{c}{$\begin{array}{c}\text { Estadístico } \\
\text { Bera-jarque }\end{array}$} & ADF & PP & KPSS \\
\hline Libex35 & 9,189 & 0,230 & $-0,129$ & 2,659 & 9,677 & 8,588 & 25,049 & $-1,877$ & $-1,726$ & 1,606 \\
Rendimiento & $-0,006$ & 1,535 & 0,088 & 8,323 & 13,484 & $-9,587$ & 3881,010 & $-11,137$ & $-69,430$ & 0,479 \\
\hline
\end{tabular}

Nota: Los valores críticos de los contraste de ADF y PP son $-3,43,-2,86$ y $-2,57$ al $1 \%, 5 \%$ y $10 \%$, respectivamente.

Los valores críticos del contraste de KPSS son 0,74, 0,45 y 0,35 al 1\%, 5\% y $10 \%$ respectivamente.

Fuente: Elaboración propia.

De los contrastes de raíz unitaria se deduce que no se puede rechazar que el Ibex35 sea I(1), frente a la alternativa de ser I(0), mientras que si se rechaza la hipótesis de I(1), frente a la alternativa de ser I(0), en el caso del rendimiento. Por su parte, la Tabla 2 recoge los coeficientes de autocorrelación de ambas series y de la volatilidad del rendimiento. Como puede observarse, los coefi-

\footnotetext{
${ }^{7}$ Se trata de un estimador insesgado de la desviación típica de los rendimientos.

${ }^{8}$ El primer estudio sobre exceso de curtosis en series financieras fue Mandelbrot (1963). Este autor observó que las variaciones de los precios generalmente presentaban distribuciones apuntadas y con colas gruesas.
} 
cientes de autocorrelación del Ibex35 son muy elevados y caen lentamente, mientras que en el caso del rendimiento la correlación, aunque muy próxima a cero, es estadísticamente distinta de cero ${ }^{9}$. Por su parte, se observa que la correlación de la volatilidad del rendimiento también es estadísticamente significativa, siempre es positiva, con valores no muy elevados que decrecen muy lentamente ${ }^{10}$. Esta evidencia indicaría la presencia de heterocedasticidad condicionada en la serie del rendimiento, de no linealidad ${ }^{11}$ o de ambos ${ }^{12}$.

Tabla 2

Autocorrelaciones muestrales

\begin{tabular}{lcccccccccc}
\hline & $\boldsymbol{\rho}=\mathbf{1}$ & $\boldsymbol{\rho}=\mathbf{2}$ & $\boldsymbol{\rho}=\mathbf{3}$ & $\boldsymbol{\rho}=\mathbf{4}$ & $\boldsymbol{\rho}=\mathbf{5}$ & $\boldsymbol{\rho}=\mathbf{6}$ & $\boldsymbol{\rho}=\mathbf{7}$ & $\boldsymbol{\rho}=\mathbf{8}$ & $\boldsymbol{\rho}=\mathbf{1 2}$ & $\boldsymbol{\rho}=\mathbf{2 4}$ \\
\hline Libex35 & 0,998 & 0,995 & 0,993 & 0,991 & 0,990 & 0,988 & 0,986 & 0,984 & 0,978 & 0,955 \\
Q-Statistic & 3273 & 6532 & 9778 & 13013 & 16237 & 19449 & 22652 & 25946 & 38517 & 75501 \\
Rendimiento & $-0,002$ & $-0,049$ & $-0,043$ & 0,022 & $-0,044$ & $-0,022$ & $-0,006$ & 0,042 & 0,019 & 0,030 \\
Q-Statistic & 0,01 & 7,75 & 13,78 & 15,41 & 21,83 & 23,37 & 23,49 & 29,31 & 40,30 & 53,33 \\
Volatilidad & 0,202 & 0,258 & 0,262 & 0,278 & 0,242 & 0,257 & 0,236 & 0,240 & 0,217 & 0,173 \\
Q-Statistic & 133,61 & 352,73 & 578,42 & 832,36 & 1025,30 & 1242,00 & 1426,00 & 1615,30 & 2275,40 & 3791,90 \\
\hline
\end{tabular}

Fuente: Elaboración propia.

Las Figuras 1 y 2 recogen la evolución temporal del índice y de su rendimiento, respectivamente. En ellos se observa que tanto la media como la varianza del Ibex35 son cambiantes en el tiempo, presentando el comportamiento típico de una serie con varianza estocástica y, por tanto, no estacionaria. Por su parte, su primera diferencia presenta un comportamiento de serie estacionaria, observándose que se mueve en torno a un nivel constante e igual a cero, aunque presenta una varianza que no es constante a lo largo del tiempo. Se observa que existen períodos de elevada volatilidad, seguidos de otros en los que la volatilidad es menor. Este tipo de comportamiento se denomina agrupamiento de la volatilidad o clustering de volatilidad. La evidencia de volatilidad variable en el tiempo ya fue remarcada por Mandelbrot (1963), quien observaba que los rendimientos de los mercados bursátiles presentaban grandes variaciones seguidas

\footnotetext{
${ }^{9}$ La evidencia empírica muestra que muchas series temporales financieras presentan nula o escasa estructura regular en la dinámica de la media, siendo suficientemente representada por modelos AR(1) ó MA(1) con parámetros pequeños (Afonso y Pérez, 2001, pp.58).

${ }^{10}$ Mismo resultado se obtiene si en lugar de esta definición de la volatilidad se considera el cuadrado de los rendimientos.

${ }^{11}$ Olmedo et al., (2007) evidencian la presencia de no linealidad en el proceso generador de los rendimientos del Ibex35 para el período comprendido entre el 1 de abril de 1990 y 10 de agosto de 2002.

${ }^{12}$ Pavlidis et al., (2010) y Blake and Kapetanios (2007).
} 
de otras grandes variaciones, de uno u otro signo, mientras que pequeños cambios eran seguidos por pequeños cambios.

Figura 1

Índice Ibex35

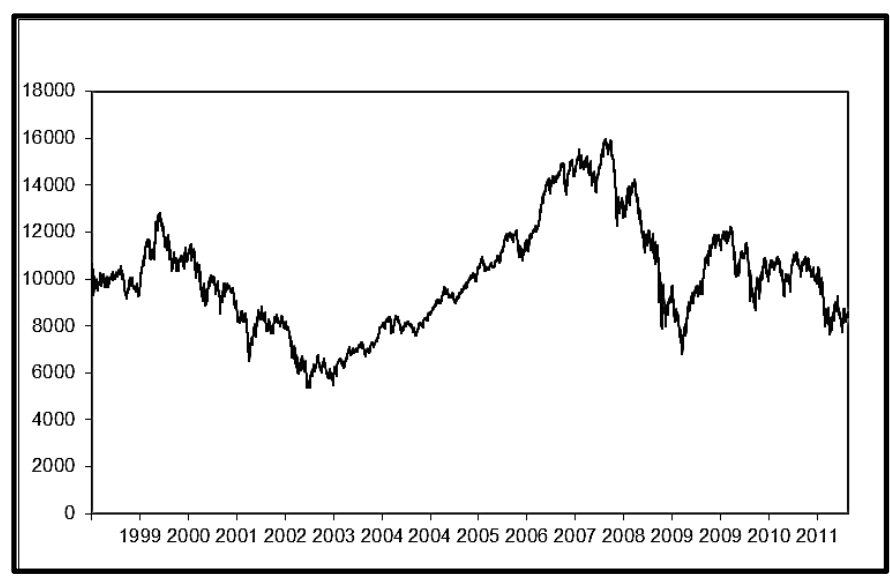

Fuente: Boletín estadístico del Banco de España.

Figura 2

Rendimiento del Ibex35

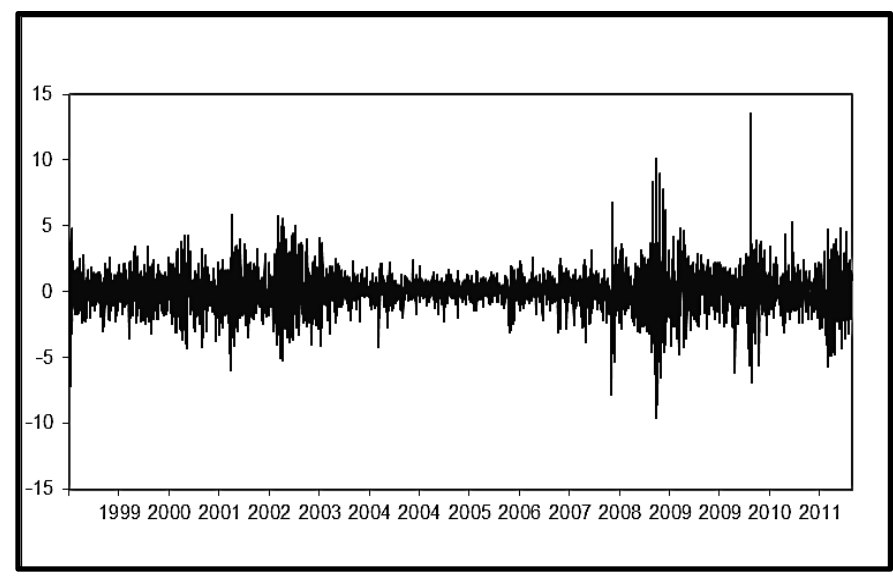

Fuente: Elaboración propia a partir de los datos del Boletín estadístico del Banco de España.

Finalmente, en la Figura 2 se observan algunos valores muy elevados, tanto positivos como negativos, que hacen pensar en la presencia de observaciones atípicas u outliers. La Figura 3 presenta la serie normalizada del rendimiento 
con el objetivo de revelar la existencia de outliers en la serie. Como puede advertirse, existen varias observaciones que pueden considerarse outliers por superar en más de tres veces la desviación típica de la serie normalizada. El mayor número de estas observaciones se concentran en el año 2008, con diecisiete observaciones, 2002 con ocho y 2011 con siete. En 2010 se detectan cinco, tres en 2009 y dos en 2001 y $1999^{13}$. De las 3.285 observaciones, 45 de ellas presentan estas características, lo que representa el $1,37 \%$ de las observaciones ${ }^{14}$. Estas observaciones coinciden, como era de esperar, con acontecimientos extraordinarios, como el ocurrido en septiembre de 2001, y con períodos de elevada incertidumbre en los mercados financieros. Estos rendimientos tan elevados aparecen con mayor probabilidad de lo que cabría esperar en una distribución normal, lo que genera la presencia observada de colas anchas en su distribución.

Figura 3

Rendimiento normalizado del Ibex35

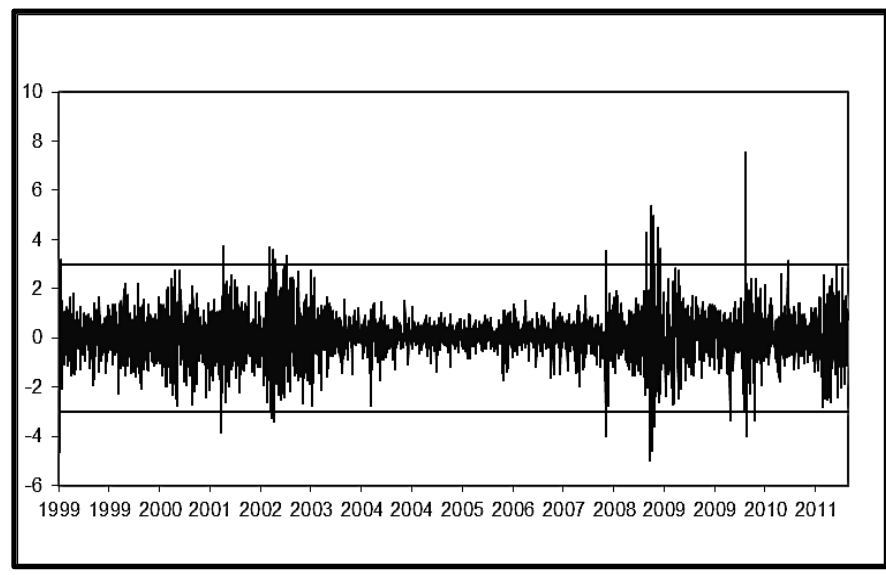

Fuente: Elaboración propia a partir de los datos del Boletín estadístico del Banco de España.

${ }^{13}$ En concreto, estas observaciones corresponden al 13 y 18 de enero de 1999, al 11 y 24 de septiembre de 2001, al 5, 15, 19, 25, 29, 30 de julio, al 8 de agosto y 15 de octubre de 2002, al 21, 23, 24 de enero, 5 de febrero, 19 de septiembre, 6, 8, 10, 13, 15, 22, 24, 29 de octubre, 4, 6, 24 de noviembre y 8 de diciembre de 2008, al 2, 5, 10 de marzo de 2009, al 4 de febrero, 4, 10, 14 de mayo y 29 de junio de 2010 y, finalmente, al 12 de enero, 10, 12, 18 de agosto, 5, 22 de septiembre y 27 de octubre de 2011.

${ }^{14}$ Se ha considerado también el método de los cuartiles para detectar outliers y este método, para un valor crítico de 2,5, identifica los mismos outliers, a excepción del 11 de septiembre de 2001, 15 de julio de 2002, 23 de enero de 2008 y 5 de marzo de 2009. En estas cuatro observaciones el rendimiento es positivo y en ningún caso supera la cota del $4,7 \%$. Si el valor crítico se fija en 3, el número de outliers cae a 22. 


\section{METODOLOGÍA ECONOMÉTRICA: ESPECIFICACIÓN Y RESULTADOS}

Con el objetivo de recoger todas y cada una de las características observadas en la serie se va a proceder a especificar un modelo lo más flexible posible, de tal forma que permita contemplar la posible no linealidad de la serie, a la vez que tenga en cuenta la presencia de heterocedasticidad condicional. De esta forma, el modelo STAR de Tong $(1978)^{15}$ resulta útil para modelizar la no linealidad en la media condicional de las series temporales ${ }^{16}$. Por otro lado, los modelos con varianza heterocedástica condicional autoregresiva (ARCH) y sus extensiones ${ }^{17}$ (GARCH, EGARCH, TGARCH y APARCH) permiten recoger el exceso de curtosis y el clustering de volatilidad. Por esta razón, Tong (1990) sugirió el modelo STAR con varianza heterocedástica condicional autoregresiva. Por su parte, muchos autores han mostrado que las series financieras presentan una varianza condicional asimétrica ${ }^{18}$, por lo que el modelo STAR se puede ampliar con varianza heterocedástica condicional que incorpore la existencia de asimetría en la volatilidad de las series. En esta línea, Li y Li (1996) propusieron un double-threshold autoregressive heteroscedastic model, es decir un modelo autoregresivo con efecto umbral tanto en la media como en la varianza condicional.

En este trabajo se va a estimar un modelo STAR-APARCH, esto es un STAR de tres regímenes con varianza heterocedástica condicional asimétrica. Este modelo viene especificado de la siguiente forma:

$$
R_{t}=\varepsilon_{t}+\left\{\begin{array}{lr}
\mu_{1}+\sum_{i=1}^{p} \alpha_{1 i} R_{t-i} & R_{t-d}>\lambda \\
\mu_{2}+\sum_{i=1}^{p} \alpha_{2 i} R_{t-i} & -\lambda \leq R_{t-d} \leq \lambda \\
\mu_{3}+\sum_{i=1}^{p} \alpha_{3 i} R_{t-i} & R_{t-d}<-\lambda
\end{array}\right.
$$

donde $R_{t}$ es el rendimiento en $\mathrm{t}$ definido por la ecuación (1) y la variable transición es $R_{t-d}$ con un retardo d desconocido. Se supone que el umbral, $\lambda$, es simétrico alrededor de cero y desconocido. El término de error puede descomponerse como:

\footnotetext{
${ }^{15}$ Propuesto inicialmente por Tong (1978) y posteriormente discutido con detalle por Tong and Lim (1980) y Tong (1983).

${ }^{16}$ Li y Li (1996).

${ }^{17}$ De Arce (2004) hace un repaso de las principales especificaciones de los modelos ARCH a lo largo del tiempo.

${ }^{18}$ Schwert (1989), Nelson (1991), Rabemananjara y Zakoian (1993).
} 


$$
\varepsilon_{t}=\sigma_{t} v_{t} \quad v_{t} \sim \operatorname{iid}(0,1)
$$

siendo $\sigma_{t}^{2}=E\left(\varepsilon_{t}^{2} / I_{t-1}\right)$ la varianza condicional del error, dado el conjunto de información disponible en $\mathrm{t}-1, I_{t-1}, \mathrm{y} E\left(\varepsilon_{t} / I_{t-1}\right)=0$. Por tanto, el término de error no presenta correlación pero su varianza condicional cambia a lo largo del tiempo.

Asimismo, se va a considerar la existencia de efectos asimétricos de la varianza condicional a las innovaciones pasadas, quedando especificada de la siguiente forma ${ }^{19,20}$ :

$$
\sigma_{t}^{\delta}=\alpha_{0}+\sum_{i=1}^{p} \alpha_{i}\left(\left|\varepsilon_{t-i}\right|-\gamma \varepsilon_{t-i}\right)^{\delta}+\sum_{j=1}^{q} \beta_{j} \sigma_{t-j}^{\delta}
$$

donde $\alpha_{0}>0, \alpha_{i} \geq 0 \forall i, \beta_{j} \geq 0 \forall j, \delta \geq 0$ y $|\gamma| \leq 1$. Para que el proceso sea estacionario ha de cumplirse que: $\sum_{i=1}^{p} \alpha_{i} k_{i}+\sum_{j=1}^{q} \beta_{j}<1, \quad k_{i}=E\left(\left|v_{t}\right|-\gamma v_{t}\right)^{\delta}$. El parámetro $\gamma_{i}$ recoge el efecto leverage ${ }^{21}$, de tal forma que si es igual a cero no existe tal efecto y los shocks positivos tienen el mismo efecto que los negativos.

El modelo (3) puede reescribirse, tal y como hace Hansen (2000), en una simple ecuación como:

$$
R_{t}=I_{t}\left\{\mu_{1}+\sum_{i=1}^{p} \alpha_{1 i} R_{t-i}\right\}+\left(1-I_{t}-J_{t}\right)\left\{\mu_{2}+\sum_{i=1}^{p} \alpha_{2 i} R_{t-i}\right\}+J_{t}\left\{\mu_{3}+\sum_{i=1}^{p} \alpha_{3 i} R_{t-i}\right\}+\varepsilon_{t}
$$

donde $I_{t}$ y $J_{t}$ son dos funciones indicadores definidas de la siguiente forma:

$$
I_{t}=\left\{\begin{array}{ll}
1 & \text { si } R_{t-d}>\lambda \\
0 & \text { si } R_{t-d} \leq \lambda
\end{array}\right\} \quad J_{t}=\left\{\begin{array}{ll}
1 & \text { si } R_{t-d}<-\lambda \\
0 & \text { si } R_{t-d} \geq-\lambda
\end{array}\right\}
$$

${ }^{19}$ Se consideró que la especificación del término de error recogería, como así se refleja en los resultados, adecuadamente el comportamiento observado de la volatilidad del rendimiento. No obstante, en lugar de considerar un término de error común a los tres regímenes, podría especificarse un proceso más complejo, contemplando la posibilidad de tres regímenes diferentes en la volatilidad del término de error, pero esto supera en todo caso el objetivo de este trabajo.

${ }^{20}$ Ding et al., (1993) propusieron el modelo APARCH, que contempla impactos asimétricos en la volatilidad, lo que permite recoger que los movimientos a la baja en el mercado de capitales generan mayores volatilidades que los movimientos al alza. Asimismo, este modelo, probablemente, es el más interesante de los modelos tipo $\mathrm{ARCH}$, puesto que es un modelo muy general que incluye, como casos especiales, a los modelos ARCH, GARCH y TGARCH.

${ }^{21}$ El efecto leverage hace referencia a la existencia de correlación negativa entre los shocks pasados y la volatilidad. Si el coeficiente $\gamma_{i}$ es positivo, los shocks negativos tienen mayor efecto en la volatilidad que los positivos. 
En la estimación del modelo (6) se ha considerado que el retardo d puede tomar valores desde 1 a 5 . Por su parte, el valor que puede tomar el parámetro umbral estará incluido en el intervalo $[\underline{\lambda} \bar{\lambda}]$. Para determinar los valores extremos del intervalo, se ordena de forma creciente las observaciones de $R_{t}$ en valor absoluto, y se selecciona el valor mínimo del umbral $\underline{\lambda}$ de forma que el $15 \%$ de las observaciones de $\left|R_{t}\right|$ se sitúen por debajo de él. El valor máximo del umbral $\bar{\lambda}$ vendrá determinado por aquel valor para el cual el $15 \%$ de las observaciones de $\left|R_{t}\right|$ estén situadas por encima de él. De esta forma se garantiza que al menos el $15 \%$ de las observaciones de $R_{t}$ se encuentran dentro o fuera de la banda, evitando que la estimación esté influida por unos pocos outliers.

El número de retardos considerados $\left(\Delta R_{t-i}\right)$ para describir la dinámica no han sido superior a tres ${ }^{22}$. Para cada retardo, los valores óptimos del umbral y del retardo d se obtienen estimando la ecuación (6) para diferentes valores de estos parámetros y seleccionando el par para el que el criterio de información de Akaike (AIC) es más pequeño. Más específicamente, para un valor fijo de d se selecciona aquel valor de $\lambda$ para el cual el AIC es más pequeño:

$$
\begin{gathered}
\hat{\lambda}(d)=\min \\
\lambda \in[\lambda C(d, \lambda) \\
\lambda=[\bar{\lambda}]
\end{gathered}
$$

Posteriormente, se selecciona el valor de d para el cual nuevamente el AIC toma el valor más reducido:

$$
\hat{d}=\min \operatorname{AIC}(\hat{\lambda}(d))
$$

Este proceso se hace para cada retardo considerado, de tal forma que el modelo finalmente elegido se selecciona entre los tres mejores modelos previamente seleccionados en términos de $\mathrm{d}$ y $\lambda$. Procediendo de esta forma, el número de retardos que describe la dinámica es de dos, el retardo de la variable transición es uno y el umbral $\lambda$ es igual a 0,392 . La Tabla 3 recoge los resultados del modelo STAR-APARCH estimado y de los contrastes de hipótesis realizados.

En primer lugar, se quiere hacer notar que se rechaza la hipótesis de igualdad de parámetros en los tres regímenes, por lo que el comportamiento de los rendimientos es no lineal. El número de observaciones en la banda umbral representan el $27 \%$ de las observaciones, siendo de alrededor del $36 \%$ en los regímenes situados por encima y por debajo de la banda umbral. Con respecto a

${ }^{22}$ Atendiendo al principio de parsimonia de la metodología Box-Jenkins. 
la estimación de la media condicional, puede observarse que por encima de la banda umbral el rendimiento se comporta como ruido blanco, lo que implica que el índice Ibex35 sigue un proceso paseo aleatorio. Sin embargo, dentro de la banda umbral, esto es cuando el rendimiento oscila alrededor de $\pm 0,392$, el rendimiento sigue un proceso autoregresivo de orden uno, AR(1), por lo que el Ibex35 sigue un proceso ARIMA $(1,1,0)$. El valor del coeficiente estimado indica que, dentro de la banda, el rendimiento ajusta en un $40 \%$ el rendimiento obtenido en el período anterior. El signo negativo del coeficiente estimado evidencia la existencia de correlación serial negativa en el rendimiento, tal y como han evidenciado muchos otros autores a partir de los trabajos seminales de Fama y French (1988) y Poterba y Summers (1988). La evidencia de correlación serial negativa en los rendimientos implica el rechazo de la HME. Finalmente, por debajo de la banda umbral, es decir, cuando el rendimiento se sitúa por debajo de $-0,392$, éste ajusta en un $10 \%$ el rendimiento obtenido en los dos períodos anteriores. Ello significa que el rendimiento se comporta como un $\operatorname{AR}(2)^{23}$, por lo que el Ibex35 sigue un proceso $\operatorname{ARIMA}(2,1,0)$. Estos resultados implican que por encima de la banda la nueva información se refleja completamente en el precio de los activos bursátiles, pero no así por debajo y dentro de la banda umbral.

Los resultados obtenidos con el modelo estimado permiten identificar como se comporta el mercado en períodos con tendencia alcista (bull market), con tendencia bajista (bear market) y con tendencia lateral. En particular, se ha obtenido que cuando el mercado está obteniendo rentabilidades por encima de la banda se comporta como un mercado eficiente. Esto se correspondería con un mercado con índices en positivo, que hace que los inversores se muestren confiados y respondan de forma eficiente a las noticias, dando lugar a que no existan posibilidades de ganancias por encima de la normal. Sin embargo, por debajo de la banda, los índices bursátiles están cayendo por lo que se pierde confianza en el mercado. En este contexto, los inversores se centraran en tratar de reducir sus pérdidas, de tal forma, que en muchas ocasiones, hacen caso omiso de las noticias. Este comportamiento implica ineficiencia del mercado. Por su parte, dentro de la banda, los índices no suben ni bajan significativamente, sino que se mantienen acotados entre un mínimo y un máximo, dando lugar a lo que se conoce como tendencia lateral. En estos períodos, los datos indican que el mercado se comporta de forma muy ruidosa, y el rendimiento cruza constantemente su media, alternando observaciones sucesivas a diferentes lados de la media, tal y como corresponde al comportamiento de un AR(1) con coeficiente negativo.

Se ha comprobado que los residuos del modelo estimado se compartan ade-

${ }^{23}$ Las raíces son complejas, con parte real igual a 0 y parte imaginaria igual a $\pm 0,316 i$. 
cuadamente de acuerdo a una batería de contrastes a los que se les ha sometido y que aparecen recogidos en la Tabla 3. Así, no se pueden rechazar ni la hipótesis de no autocorrelación ni la de homocedásticidad de los residuos.

Tabla 3

Estimación model STAR-APARCH

\begin{tabular}{lcccc}
\hline & & $\boldsymbol{R}_{\boldsymbol{t}-\mathbf{1}}>\boldsymbol{\lambda}$ & $-\lambda \leq \boldsymbol{R}_{\boldsymbol{t}-\mathbf{1}} \leq \boldsymbol{\lambda}$ & $\boldsymbol{R}_{\boldsymbol{t}-\mathbf{1}}<-\lambda$ \\
\hline Constante & & $-0,013$ & 0,014 & 0,005 \\
& & $(-0,263)$ & $(0,419)$ & $(0,078)$ \\
$\mathrm{R}_{\mathrm{t}-1}$ & & 0,032 & $-0,403$ & 0,008 \\
& & $(0,808)$ & $(-2,923)$ & $(0,158)$ \\
$\mathrm{R}_{\mathrm{t}-2}$ & & 0,024 & 0,002 & $-0,101$ \\
& & $(0,978)$ & $(0,057)$ & $(-2,854)$ \\
\hline Observaciones & & 1232 & 889 & 1162 \\
$\lambda$ & 0,392 & & & \\
$\mathrm{R}^{2}$ & 0,005 & & & \\
$\mathrm{SE}$ & 1,534 & & & \\
DW & 2,002 & & & \\
\hline Residuos: & & & \\
$\mathrm{Q}(1)$ & 0,855 & & \\
$\mathrm{Q}(12)$ & 0,647 & & \\
$\mathrm{Q}(24)$ & 0,908 & & & \\
\hline Residuos & & & & \\
$\mathrm{Q}(1)$ & 0,108 & & & \\
$\mathrm{Q}(12)$ & 0,167 & & & \\
$\mathrm{Q}(24)$ & 0,303 & & & \\
\hline
\end{tabular}

$\mathrm{H}_{0}$ : Igualdad de parámetros 3 regímenes

$p$-value $\quad 0,008$

Estimación varianza condicional:

$\mathrm{H}_{0}: \gamma=1$

$$
\sigma_{t}^{1.104}=0.027+0.071\left(\left|\hat{\varepsilon}_{t-1}\right|-0.903 \hat{\varepsilon}_{t-1}\right)^{1.104}+0.920 \sigma_{t-1}^{1.104}
$$

$p$-value

0,615

$\mathrm{H}_{0}: \delta=1$

p-value $\quad 0,495$

$\mathrm{H}_{0}: \gamma=\delta=1$

p-value

0,661

Nota: Los valores entre paréntesis son los valores del estadístico $t$ de Student calculados con errores estándar consistentes bajo heteroscedasticidad.

SE es el error estándar de la regresión y $Q$ (.) hace referencia al estadístico $Q$ de Ljung-Box para los retardos indicados dentro del paréntesis. Sus valores corresponden al p-value.

Fuente: Elaboración propia.

Con respecto a la volatilidad condicional estimada, el parámetro que recoge 
el efecto leverage es significativo e igual a uno. Este resultado tiene dos implicaciones, una la existencia de efecto leverage y la segunda es que, al ser el parámetro igual a uno, las noticias positivas no afecta a la volatilidad, mientras que si que lo hacen las noticias negativas, que la aumentan. Este resultado sería acorde con la propia evidencia que permite observar que en momentos críticos, de elevada incertidumbre en los mercados, las noticias negativas aumentan considerablemente la volatilidad de los mercados mientras que las noticias positivas apenas les afecta. Por su parte, en períodos de estabilidad bursátil, el rumor de una noticia negativa aumenta inmediatamente la volatilidad del mercado mientras que no lo hacen las noticias positivas. Por otro lado, también se evidencia que no puede rechazarse la hipótesis de que la potencia, el parámetro $\delta$, es igual a uno ${ }^{24}$. La desviación oscila alrededor de un $2,7 \%$ diario, siendo su valor final el $92 \%$ de la desviación del día anterior y el $14 \%$ de las noticias negativas del día anterior ${ }^{25}$, puesto que si son positivas no tienen, como se ha comentado, impacto alguno. Por su parte, la volatilidad no condicional o a largo plazo diaria es igual a $1,1^{26}$.

Finalmente, se compara la capacidad predictiva del modelo STAR-APARCH con el modelo paseo aleatorio, así como con un modelo lineal en media ${ }^{27}$. Para ello se evalúa la capacidad predictiva de los modelos dentro y fuera del período muestral, considerándose como período de predicción extramuestral desde el 2 de enero al 31 de mayo de 2012. La Tabla 4 recoge los estadísticos que permiten analizar la capacidad predictiva de los modelos. Para el período completo ${ }^{28}$, los resultados indican que las diferencias, aunque pequeñas, están a favor del modelo STAR-APARCH. El test de Dielbold y Mariano ${ }^{29}$ confirman estos resultados al obtener estadísticos negativos y significativos en ambos $\operatorname{casos}^{30}$. Al analizar el índice de Theil se comprueba que es menor en el modelo STAR-

${ }^{24} \mathrm{Si} \delta=1$ el modelo es el modelo generalizado TARCH de Zakoian (1994).

${ }^{25}$ Se ha comprobado que la volatilidad condicional cumple con los requisitos de estacionariedad puesto que $0.071 k_{1}+0.92<1, \quad k_{1}=E\left(\left|v_{t}\right|-\gamma v_{t}\right)=0.79$

${ }^{26}$ Se obtiene de $\frac{\alpha_{0}}{\left(1-\alpha_{1} k_{1}-\beta_{1}\right)}=\frac{0.027}{1-0.071 k_{1}-0.92}$, siendo $k_{1}=E\left(\left|v_{t}\right|-\gamma v_{t}\right)=0.79$

${ }^{27}$ El modelo ARMA(1,1)-APARCH ha sido, según el criterio de Schwarz, el que mejor se ha ajustado a los datos.

${ }^{28}$ Dado que el período completo incluye el período muestral y extramuestral, los resultados pueden interpretarse en términos de bondad de ajuste a los datos más que como capacidad predictiva. Asimismo, indicar que también se analizó la predicción desde julio de 2007 hasta el final de la muestra, pare ver el comportamiento del modelo durante la actual crisis financiera, pero dado que se obtuvieron resultados similares a los presentados se optó por no incluirlos por motivos de espacio.

${ }^{29}$ Este test contrasta la significatividad de las diferencias entre los errores.

${ }^{30} \mathrm{El}$ nivel de confianza considerado en los contrastes ha sido del $90 \%$. 
APARCH que en los otros dos modelos considerados $\mathrm{y}$, además, presenta un componente aleatorio mucho mayor.

Tabla 4

Evaluación capacidad predictiva

\begin{tabular}{|c|c|c|c|}
\hline \multicolumn{4}{|c|}{ Período: Enero 1999-Mayo 2012} \\
\hline & STAR-APARCH & Paseo aleatorio & ARMA-APARCH \\
\hline Error cuadrático medio & 0,015 & 0,015 & 0,015 \\
\hline Error absoluto medio & 0,011 & 0,011 & 0,011 \\
\hline Coeficiente de Theil & 0,930 & 0,997 & 0,972 \\
\hline Componente Sesgo & $3,1 E-04$ & $4,0 E-05$ & $1,8 E-05$ \\
\hline Componente varianza & 0,865 & 0,999 & 0,942 \\
\hline Componente aleatorio & 0,134 & $1,6 E-04$ & 0,057 \\
\hline \multicolumn{4}{|l|}{ Dielbold and Mariano test } \\
\hline ABS loss & & $-3,78 E-05$ & $-3,37 E-05$ \\
\hline ( $p=$ value $)$ & & $(0,048)$ & $(0,106)$ \\
\hline Squared loss & & $-1,21 E-06$ & $-1,56 E-06$ \\
\hline ( $p=$ value $)$ & & $(0,096)$ & $(0,0646)$ \\
\hline \multicolumn{4}{|c|}{ Período: Enero 2012-Mayo 2012} \\
\hline & STAR-APARCH & Paseo aleatorio & ARMA-APARCH \\
\hline Error cuadrático medio & 0,016 & 0,016 & 0,016 \\
\hline Error absoluto medio & 0,013 & 0,013 & 0,013 \\
\hline Coeficiente de Theil & 0,920 & 0,997 & 0,966 \\
\hline Componente Sesgo & 0,037 & 0,031 & 0,033 \\
\hline Componente varianza & 0,834 & 0,969 & 0,921 \\
\hline Componente aleatorio & 0,128 & 0,001 & 0,045 \\
\hline \multicolumn{4}{|l|}{ Dielbold and Mariano test } \\
\hline ABS loss & & $1,10 E-04$ & $1,38 E-04$ \\
\hline ( $p=$ value $)$ & & $(0,411)$ & $(0,298)$ \\
\hline Squared loss & & $-3,39 E-06$ & $-1,75 E-06$ \\
\hline ( $p=$ value $)$ & & $(0,471)$ & $(0,693)$ \\
\hline
\end{tabular}

Fuente: Elaboración propia.

Cuando el análisis se circunscribe al período extramuestral, las diferencias en los errores son muy pequeñas, de tal forma que el test de Dielbold y Mariano evidencia que no son significativas. Con respecto al índice de Theil, éste es nuevamente más pequeño en el modelo STAR-APARCH frente a los otros dos modelos, y además el peso del componente aleatorio es notablemente mayor. Estos resultados evidencian que el modelo STAR-APARCH permite predecir los rendimientos del Índice Ibex35, por lo que no se cumple la versión débil de la HME y, por tanto, la nueva información no se refleja completamente en el precio de los activos. 
Asimismo, también se evalúa la predicción de la volatilidad del rendimiento dentro y fuera de la muestra con el modelo STAR-APARCH y con los otros dos modelos. En finanzas hay interés en modelizar y predecir la volatilidad de los rendimientos de los activos, puesto que ésta puede ser considerada como una medida del riesgo de la inversión y, por tanto, es una información esencial a la hora de tomar decisiones. Siguiendo a Franses y Dijk (1996) para evaluar la habilidad en la predicción de la volatilidad del rendimiento se necesita una medida de la ésta ${ }^{31}$. En este trabajo se considera la volatilidad obtenida en el segundo apartado a través de la ecuación (2). De esta forma, el error de predicción, $u_{t}$, con el modelo STAR-APARCH vendrá dado por la expresión:

$$
u_{t}=\sigma_{t}-\hat{\sigma}_{t}
$$

donde $\sigma_{t}$ es el estimador insesgado de la volatilidad obtenido de la ecuación (2) y $\hat{\sigma}_{t}$ es la volatilidad estimada obtenida a partir de la ecuación (5).

Para comparar la predicción del modelo STAR-APARCH con una predicción ingenua, Franses y Dijk (1996) consideran el error de predicción del modelo paseo aleatorio en el cual $\hat{\sigma}_{t}=\sigma_{t-1}$. Asimismo, se ha considerado también la predicción con el modelo lineal en media ARMA-APARCH. La Tabla 5 recoge los estadísticos descriptivos para evaluar la capacidad predictiva de la volatilidad de los modelos. De los resultados de dicho cuadro se evidencia que el modelo STAR-APARCH predice mucho mejor que el modelo paseo aleatorio la volatilidad de los rendimientos, tanto en el período completo como en el período extramuestral. Sin embargo, al comparar el modelo STAR-APARCH con el modelo ARMA-APARCH las diferencias que se observan son muy pequeñas.

Se puede por tanto concluir que el modelo STAR-APARCH predice mucho mejor que el modelo aleatorio el comportamiento, tanto de la media como de la varianza condicional, del Ibex35. Esta evidencia conlleva el rechazo de la versión débil de la $\mathrm{HME}^{32}$. Asimismo, el modelo STAR-APARCH predice mucho mejor que el modelo ARMA-APARCH la media condicional de la serie de rendimientos, no observándose, como era de esperar, diferencias significativas en la predicción de la volatilidad. Ello indica que si se utiliza un modelo APARCH lineal en media para modelizar el proceso generador de los rendimientos, la volatilidad quedaría bien especificada pero no así la media condicional que presenta, como se ha evidenciado, un comportamiento no lineal y asimétrico.

${ }^{31}$ Para estos autores la volatilidad viene determinada por el cuadrado de la diferencia entre el rendimiento y su media de los últimos cuatro años.

${ }^{32}$ Pérez-Rodriguez et al., (2005) obtienen resultados que también evidencian que el paseo aleatorio no describe razonablemente el comportamiento del índice Ibex35. 
Tabla 5

Evaluación capacidad predictiva de la volatilidad

\begin{tabular}{lccc}
\hline Período: Enero 1999-Mayo 2012 & & & \\
& STAR-APARCH & Paseo aleatorio & ARMA-APARCH \\
\hline Error cuadrático medio & 1,408 & 2,925 & 1,407 \\
Error absoluto medio & 0,841 & 1,182 & 0,845 \\
Coeficiente de Theil & 0,343 & 0,444 & 0,344 \\
Período: Enero 2012-Mayo 2012 & & & \\
& STAR-APARCH & Paseo aleatorio & ARMA-APARCH \\
\hline Error cuadrático medio & 1,315 & 2,729 & 1,322 \\
Error absoluto medio & 0,949 & 1,315 & 0,950 \\
Coeficiente de Theil & 0,305 & 0,410 & 0,306 \\
\hline
\end{tabular}

Fuente: Elaboración propia.

\section{CONCLUSIONES}

En este trabajo se ha modelizado el comportamiento de la media y varianza condicional del rendimiento del índice Ibex35, durante el período que abarca desde enero de 1999 a diciembre de 2011. Para ello se ha llevado a cabo una modelización del índice Ibex35 que permitía recoger todas y cada una de las características observadas en el mismo. Los resultados han permitido concluir que el Ibex35 presenta un comportamiento no lineal y asimétrico, tanto en la media como en la varianza condicional.

Por lo que respecta a la media condicional, los resultados evidencian que por encima de la banda umbral el Ibex35 sigue un proceso paseo aleatorio y, por tanto, la nueva información se refleja completamente en el precio de los activos. Sin embargo, dentro y por debajo de la banda umbral el Ibex35 sigue un proceso ARIMA, distinto al paseo aleatorio, lo que supone que la nueva información no se refleja rápidamente en el precio de los activos, tal y como implica la HME. Por tanto, la evidencia empírica permite rechazar la versión débil de la HME. Estos resultados darían la razón a los autores que han puesto en entredicho la eficiencia de los mercados de valores y han responsabilizado a esta hipótesis de la actual crisis financiera, al permitir justificar la no intervención en los mercados.

Con respecto a la volatilidad condicionada, los resultados muestran la existencia de efecto leverage, y en concreto las noticias positivas no afectan a la volatilidad mientras que las noticias negativas la aumentan, con el consiguiente impacto sobre el riesgo asociado a las inversiones. 


\section{REFERENCIAS BIBLIOGRÁFICAS}

AFONSO, J.A. y PÉREZ, B. (2001). "Influencia de la estructura heterocedástica en la diversificación de carteras de acciones". Estudios de Economía Aplicada, 17, pp. 53-68.

BALI, T.G.; DEMIRTAS, K.O. y LEVY, H.(2008). "Nonlinear mean reversion in stock prices". Journal of Banking and Finance, 32, pp. 767-782.

BALL, R. (2009). "The global financial crisis and the efficient market hypothesis: What have we learned?". Journal of Applied Corporate Finance, 21, pp. 816.

BLAKE, A.P. y KAPETANIOS, G. (2007). "Testing for ARCH in the presence of nonlinearity of unknown form in the conditional mean". Journal of Econometrics, 137, pp. 472-488.

BORGES, M.R. (2011). "Efficient market hypothesis in European stock markets". European Journal of Finance, 16(7), pp. 711-726.

CAO, C. y TSAY, R.S. (1992). "Nonlinear time-series analysis of stock volatility". Journal of Applied Econometrics, 7, pp. 165-185.

CHAN, K.C.; GUP, B.E. y PAN, M.S. (1997). "International stock market efficiency and integration: A study of eighteen nations". Journal of Business Finance and Accounting, 24, pp. 803-813.

CHAUDHURI, K. y WU, Y. (2003). "Random walk versus breaking trend in stock prices: evidence from emerging markets". Journal of Banking and Finance, 27, pp. 575-592.

CHOUDHRY, T. (1997). "Stochastic trends in stock prices: evidence from Latin American markets". Journal of Macroeconomics, 19, pp. 285-304.

DE ARCE, R. (2004). "20 años de modelos ARCH: una visión de conjunto de las distintas variantes de la familia". Estudios de Economía Aplicada, 22(1), pp. 1-27.

DE BONDT, W.F.M. y THALER, R. (1985). "Does the stock market overreact?". Journal of Finance, 40, pp. 793-805.

DING, Z.; ENGLE, R.F. y GRANGER, C.W.J. (1993). "A long memory property of stock market returns and a new model". Journal of Empirical Finance, 1, pp. 83-106.

FAMA, E.F. (1970). "Efficient capital markets: A review of theory and empirical work". Journal of Finance, 25, pp. 383-417.

FAMA, E.F. y FRENCH, K.R. (1988). "Permanent and temporary components of stock prices" Journal of Political Economy, 96, pp. 246-273.

FRANSES, P.H. y DIJK, D.V. (1996). "Forecasting stock market volatility using (Non-linear) garch models". Journal of Forecasting, 15, pp. 229-235.

GRANTHAM, J. (2010). "What a decade!" GMO Quartely Letter. January, pp. 18. 
GRIEB, T. y REYES, M. (1999). "Random walk tests for Latin American equity indexes and individual firms". Journal of Financial Research, 22(4), pp. 371383

HANSEN, B.E. (2000). "Sample splitting and threshold estimation". Econometrica, 68, pp. 575-603.

HUBER, P. (1997). "Stock market returns in thin markets: evidence from the Vienna stock exchange". Applied Economics Letters, 7, pp. 493-498.

JAMES, H. (2009): The creation and destruction of value. The globalization cycle. USA: Harvard University Press.

JENSEN, M.C. (1978): "Some anomalous evidence regarding market efficiency" Journal of Financial Economics, 6, pp. 95-101.

KAWAKATSU, K. y MOREY, M.R. (1999). "An empirical examination of financial liberalization and the efficiency of the emerging market stock prices". Journal of Financial Research, 22, pp. 358-411.

KIM, J. y SHAMSUDDIN, A. (2009). "Short-horizon return predictability in international equity markets". Working paper 1, La Trobe University.

KIM, J.H.; SHAMSUDDIN, A. y LIM, K.P. (2011). "Stock return predictability and the adaptive markets hypothesis: Evidence from century-long US data". Journal of Empirical Finance, 18, pp. 868-879.

LEE, C.C; LEE, J.D. y LEE, C.C. (2010). "Stock prices and the efficient market hypothesis: Evidence from a panel stationary test with structural breaks". Japan and the World Economy, 22, pp. 49-58.

LI, C.W. y LI, W.K. (1996). "On a double-threshold autoregressive heteroskedastic time series model". Journal of Applied Econometrics, 11, pp. 253-274.

LIM, K.P. (2009). "Weak-form market efficiency and nonlinearity: evidence from Middle East and African stock indices". Applied Economics letters, 16(5), pp. 519-522.

LIM, K.P. y BROOKS, R.D. (2009). "Are Chinese stock markets efficient? Futher evidence from a battery of nonlinearity tests". Applied Financial Economics, 16, pp. 147-155.

LIM, K.P.; Brooks, R.D. y Hinich, M.L. (2008). "Nonlinear serial dependence and the weak-form efficiency of Asian emerging stock markets". Journal of International Financial Markets, Institutions and Money, 18(5), pp. 527-544.

LIU, X.; SONG, H. y ROMILLY, P. (1997). "Are Chinese stock markets efficient? A cointegration and causality analysis". Applied Economics letters, 4, pp. 511-515.

LO, A.W. y MACKINLAY, A.C. (1988). "Stock market prices do not follow random walks: Evidence from a simple specification test". Review of Financial Studies, 1, pp. 41-66.

MANDELBROT, B. (1963). "The variation of certain speculative prices". Journal of Business 36, pp. 394-419. 
NARAYAN, P.J. (2006). "The behaviour of US stock prices: Evidence from a threshold autoregressive model". Mathematics and computers in simulation, 71, pp. 103-108.

NELSON, D.B. (1991). "Conditional heteroskedasticity in asset returns: A new approach". Econometrica, 59, pp. 347-370.

OLMEDO, E.; VELASCO, F. y VALDERAS, J.M. (2005). "Caracterización no lineal y predicción no paramétrica en el IBEX35". Estudios de Economía Aplicada, 23(3), pp. 815-842.

PAVLIDIS E.G.; PAYA, I. y PEEL, D.A. (2010). "Specifying smooth transition regression models in the presence of conditional heteroskedasticity of unknown form". Studies in nonlinear dynamics and econometrics, 14: The Berkeley Electronic Press.

PÉREZ-RODRÍGUEZ, J.V. y TORRA, S. (2003). "Reversión a la media, no linealidad y cambios de régimen en la evolución temporal del lbex35". Revista Española de Financiación y Contabilidad, 32(119), pp. 1177-1203.

PÉREZ-RODRÍGUEZ, J.V.; TORRA, S. y ANDRAD-FÉLIX, J. (2005). "STAR and ANN models: forecasting performance on the Spanish "Ibex-35" stock index". Journal of Empirical Finance, 12, pp. 490-509.

PÉREZ-RODRÍGUEZ, J.V.; TORRA, S. y ANDRAD-FÉLIX, J. (2005). "Are Spanish lbex35 stock future index returns forecasted with non-linear models?". Applied Financial Economics, 15, pp. 963-975.

POTERBA, J.A. y L.M. SUMMER (1988). "Mean reversion in stock prices: Evidence and implications". Journal of Financial Economics, 22, pp. 27-59.

RABEMANANJARA, R. y ZAKOIAN, J.M. (1993). "Threshold arch models and asymmetries in volatility". Journal of Applied Econometrics, 8, pp. 31-49.

RASHID, A. y AHMAD, S. (2008). "Predicting stock returns volatility: An evaluation of linear vs. nonlinear methods". International Research Journal of Finance and Economics, 20, pp. 141-150.

SAMUELSON, P.A. (1965). "Proof that properly anticipated prices fluctuate randomly". Industrial Management Review, 6, pp. 41-49.

SEWELL, M. (2011). "History of the efficient market hypothesis". Research note, RN/11/04, UCL department of computer science.

SCHWERT, G.W. (1989). "Why does stock market volatility change over time?". Journal of Finance, American Finance Association, 44, pp. 1115-1153.

SHILLER, R.L. (1984). "Stock prices and social dynamics". Brookings Papers on Economic Activity, 2, pp. 457-510.

STIGLITZ, J.E. (2010). Freefall: America, free markets and the sinking of the world economy, USA, WW Norton and Company.

TONG, H. (1978). "On a threshold model". Pattern Recognition and Signal Processing (ed. C.H. Chen), Amsterdam: pp. 575-586.

TONG, H. (1983). "Threshold models in non-linear time series analysis". Lecture Notes in Statistics, 21, New York: Springer-Verlag. 
TONG, H. (1990). "Nonlinear time series: a dynamical system approach". Oxford University Press, Oxford.

TONG, H. y LIM, K.P. (1980). "Threshold autoregression, limit cycles and cyclical data". Journal of the Royal Statistical Society, Series B, 42, pp. 245-292.

TORRERO, A. (2011). "La crisis financiera internacional: Una revisión de algunas ideas sobre el papel de las finanzas en el sistema económico". Instituto universitario de análisis económico y social. Documento de trabajo 01/2011, Universidad de Alcalá.

URRUTIA, J. (1995). "Test of randmom walk and market efficiency for Latin American emerging markets". Journal of Financial Research, 18, pp. 299309

WOOLLEY, P. (2010). "Why are financial markets so inefficient and exploitative and a suggested remedy". En A. Turner et al.,: The future of finance: the LSE report. London School of Economics and Political Science.

WORHTINGTON, A. y HIGGS, H. (2004). "Random walk and market efficiency in European equity markets". Global Journal of Finance and Economics, 1, pp. 59-78.

ZAKOIAN, J.M. (1994). "Threshold heteroskedastic models". Journal of Economic Dynamics and Control, 18, pp. 931-955. 
